\title{
Point-of-Care Tests for Hepatitis B Are Associated with A Higher Linkage to Care and Lower Cost Compared to Venepuncture Sampling During Outreach Screenings in an Asian Migrant Population
}

\author{
Erwin $\mathrm{Ho}^{*},{ }^{\prime}$, Peter Michielsen ${ }^{*} \dagger$, Pierre Van Damme ${ }^{\ddagger}$, Margareta leven ${ }^{\ddagger}$, Irene Veldhuijzen \\ and Thomas Vanwolleghem, ${ }^{*}$, ,
}

Background: This study compares venepuncture versus point-of-care (POC) HBsAg tests on screening cost and linkage to care in prospective outreach screenings in an Asian population in three major cities in Belgium between 10/2014 and 5/2018.

Methods: Two community outreach screening programs were organised between 10/2014 and 5/2018. The first screening program used venepuncture and serologic testing for HBsAg. In the second program, $\mathrm{HBsAg}$ was tested in finger stick blood POC tests. Positive results were confirmed during outpatient visits with serologic testing. Linkage to care was defined as having received specialist care follow-up with at least one abdominal ultrasound within three months of screening.

Results: For 575 participating individuals, 571 valid results were obtained, 456 with venepuncture, and 115 using POC testing. Overall HBsAg seroprevalence was 6.8\%. Linkage to care was higher when using POC testing compared to venepuncture $(86 \%$ or $n=6 / 7$ versus $34 \%$ or $n=11 / 32 ; p=0.020)$. The POC screening program was economically more attractive with a total cost of $€ 1,461.8$ or $€ 12.7$ per person screened compared to $€ 24,819$ or $€ 54.0$ per person screened when using venepuncture testing. Results and an appointment for specialist care follow-up were given onsite with POC testing, while with venepuncture testing; results were sent within 20-45 days.

Conclusion: In an Asian migrant population in Belgium with an HBsAg seroprevalence of $6.8 \%$, HBV screening based on POC tests resulted in lower costs per person screened (76.5\% lower), and higher linkage to care (2.5 times).

\author{
Introduction \\ Viral hepatitis remains a major, worldwide health issue. \\ Recent reports from the World Health Organization [1, 2] \\ estimate that 1.34 million deaths occur yearly by viral hep- \\ atitis $B$ and $C$ infections and its sequelae. Testing guide- \\ lines and a call to eradicate chronic viral hepatitis by 2030 \\ have been recently released by the WHO, underpinned by \\ advances in diagnostics and the availability of effective \\ treatment [3, 4]. Screening for HBV is cost-effective in

\footnotetext{
* Department of Gastroenterology and Hepatology, Antwerp University Hospital, Antwerp, BE

Laboratory of Experimental Medicine and Paediatrics, University of Antwerp, Antwerp, BE

* VAXINFECTIO, Vaccine and Infectious Diseases Institute, University of Antwerp, Antwerp, BE

Division of Infectious Disease Control, Municipal Public Health Service Rotterdam-Rijnmond, Rotterdam, NL

॥ Department of Gastroenterology and Hepatology, Erasmus University Medical Centre, Rotterdam, NL

Corresponding author: Thomas Vanwolleghem

(thomas.vanwolleghem@uza.be)
}

migrant populations [10], but the linkage to care is a common challenge in these initiatives. Of all migrant populations in the European Union as a whole, individuals from Chinese descent make up the highest number of estimated HBV infected patients, with HBV seroprevalences of $6.2 \%-8.7 \%[5,7,8]$.

Screening of this hard-to-diagnose population has often been performed by community outreach methods, wherein medical staff performs screening on-site, cooperating with Chinese communities [6, 9]. The Centres for Disease Control's Hepatitis Testing and Linkage to Care (HepTLC) project showed that less than half of individuals who tested positive for HBsAg attended a first medical appointment [11]. Multiple barriers to care exist in several infectious diseases, including in hepatitis B infection care [12]. In sexually transmitted infections and HIV care, pointof-care tests (POCT) have been shown to overcome most of these barriers to care, but their impact on linkage to care in viral hepatitis B remains undetermined [13]. Recently, rapid POCT has become available for HBsAg. The performance characteristics of certain tests are excellent with sensitivities of $99.9 \%$ and specificities of $99.8 \%[14,15]$. 


\section{Methods}

This study aims to compare screening costs and linkage to care for viral hepatitis B infection in a migrant population with high expected seroprevalences using POCT or standard venepunctures during outreach screening activities.

Between 10/2014 and 5/2018, community screening programs were organised with the cooperation of Chinese community leaders, Chinese organisations and the Antwerp City Council. This allowed us to screen the same Asian population through multiple angles: during community events in various locations (churches, temples, and a local public library in Antwerp and adjacent major Belgian cities), in Asian massage parlours in Antwerp, and through opportunistic testing during mandatory integration classes.

\section{Patient and Public Involvement}

Community leaders, volunteers, and screened persons of the Chinese community all participated before, during, and after the various screening events. Preparatory meetings were organized to disseminate the rationale of the study and make arrangements for the logistics of the screening events. A pre-screening questionnaire, communication through written media, social media, and wordto-mouth were used. During screening events, community members, translators, and Antwerp City Council social workers assisted in logistics and translation services. After screening events, community members also assisted in translation during telephone calls for results and to set up outpatient appointments.

Venepuncture testing for HBsAg was subsequently performed in the Antwerp University Hospital laboratory (Elecsys HBsAg II, Roche Diagnostics $\mathrm{GmbH}$, Mannheim, Germany). POC testing for HBsAg was done with Vikia HBsAg tests (Biomérieux SA, Marcy-l'Etoile, France), according to the package insert. Testing was performed pseudonymised (no personal details were provided but results were given to the screened person) to maximize screening uptake in this difficult-to-reach population. Serologic results were given on-site after an incubation time of 15 minutes for the Vikia test, as per the package insert. Upon receiving a positive result, patients were identified and an appointment for specialist care follow-up was immediately agreed upon. POC tested patients' results were confirmed using the standard of care serologic testing (Elecsys HBsAg II, Roche Diagnostics GmbH, Mannheim, Germany) at their first outpatient visit.

Inclusion criteria were: being of Asian descent, first- or second-generation migrant and birth date before 1999, which is the year of starting universal HBV vaccination in Belgium. Excluded were individuals $<18$ years old at the screening event and third-generation migrants. Minimal clinical data were obtained after informed consent. Data obtained included: age, gender, nationality, place of birth, HBV infection status, HBV vaccination status, and previous or ongoing anti-viral treatment. Results were sent through the mail and positively screened persons were notified by phone and also invited for specialist care follow-up.

Additional reflex biochemical (ALT) and virological tests were performed on HBsAg positive samples (HBeAg and
anti-HBe antibody: Liaison, DiaSorin, Saluggia, Italy; HBV DNA: in-house method).

Screening uptake was defined as participating with the screening programs when reached (in any of the described screening events) and providing a valid serological result. Linkage to care was defined as having received specialist care follow-up within three months with HBsAg, HBeAg, anti-HBe antibody, ALT, and HBV DNA test results, and at least one abdominal ultrasound. Analysis of linkage to care was finalised three months after the last screening activity by cross-checking positively screened persons and the presence of out-patient clinic patient files at the Antwerp University Hospital. Treatment and hepatocellular carcinoma (HCC) surveillance indications were based on international clinical practice guidelines [23, 24]. All costs associated with the screening activities (personnel, screening tests, other test materials, event-related costs such as fees for screening locations, and communication costs) are listed in the euro. Tests performed in the followup were excluded, as they were not billed to the patients. Turn-around times, or the amount of time passed between venepuncture and posting of results in the venepuncture, were calculated in days.

\section{Statistics}

Before the study, a power analysis was performed to ascertain a valid sample size for the seroprevalence of HBV. Previous studies of similar scope [5-7] report a prevalence of HBV infection of $8.5-8.7 \%$. Considering estimates of the Chinese population in Belgium of approximately 13,000-23,000 individuals [16] and extrapolation of 6,239 East Asians in Antwerp city proper in 2014, a power analysis with a precision of $5 \%$ (confidence interval of $95 \%$ ) yielded a required sample size of $n=119$.

To determine differences in sampling, the seroprevalence of HBsAg between the two screening programs was compared using the Chi-square test. To compare screened persons' characteristics in terms of linkage to care, students' T-tests were used to compare continuous variables. Chi-square or, where applicable, Mid-P exact test was used to compare ordinal and categorical variables, including the association between screening programs and linkage to care. Given the absence of estimates on differences in linkage to care for both screening programs, no a priori power calculation thereof was performed.

Statistical tests were performed using SPSS version 24.0 (IBM Corp. Released 2016. IBM SPSS Statistics for Windows, Version 24.0. Armonk, NY: IBM Corp.).

\section{Results \\ Organisation of screening}

Seven events were held between 10/2014 and 12/2015 (Antwerp: $\mathrm{n}=4$; Brussels: $\mathrm{n}=2$ and Leuven $\mathrm{n}=1$ ). Both religious (two Christian fellowships/churches, a Buddhist temple) and non-religious focus groups (Hong Kongese and other Chinese societies) were involved. Subsequently, thirteen events were held between 12/2015 and 5/2018 in Antwerp, in 23 massage parlours, five events in classrooms of the Atlas Integration and Citizenship Education organization, and one time at the Antwerp University Hospital. Details are listed in Table 1. 
Table 1: Screening protocols and characteristics.

\begin{tabular}{lrl} 
Screening Protocols and Characteristics & \multicolumn{1}{c}{ Venepuncture } & Point-of-care test \\
\hline Cooperating partners & $\begin{array}{c}\text { Chinese community } \\
\text { - Key opinion leaders }\end{array}$ & $\begin{array}{c}\text { City of Antwerp/Atlas Integration } \\
\text { and Citizenship Education/Church }\end{array}$ \\
Number of hospital staff & $4-7$ & 1 \\
Number screening events & 7 & 13 \\
Number screening sites & 5 & 27 \\
Number of screened persons & 460 & 115
\end{tabular}

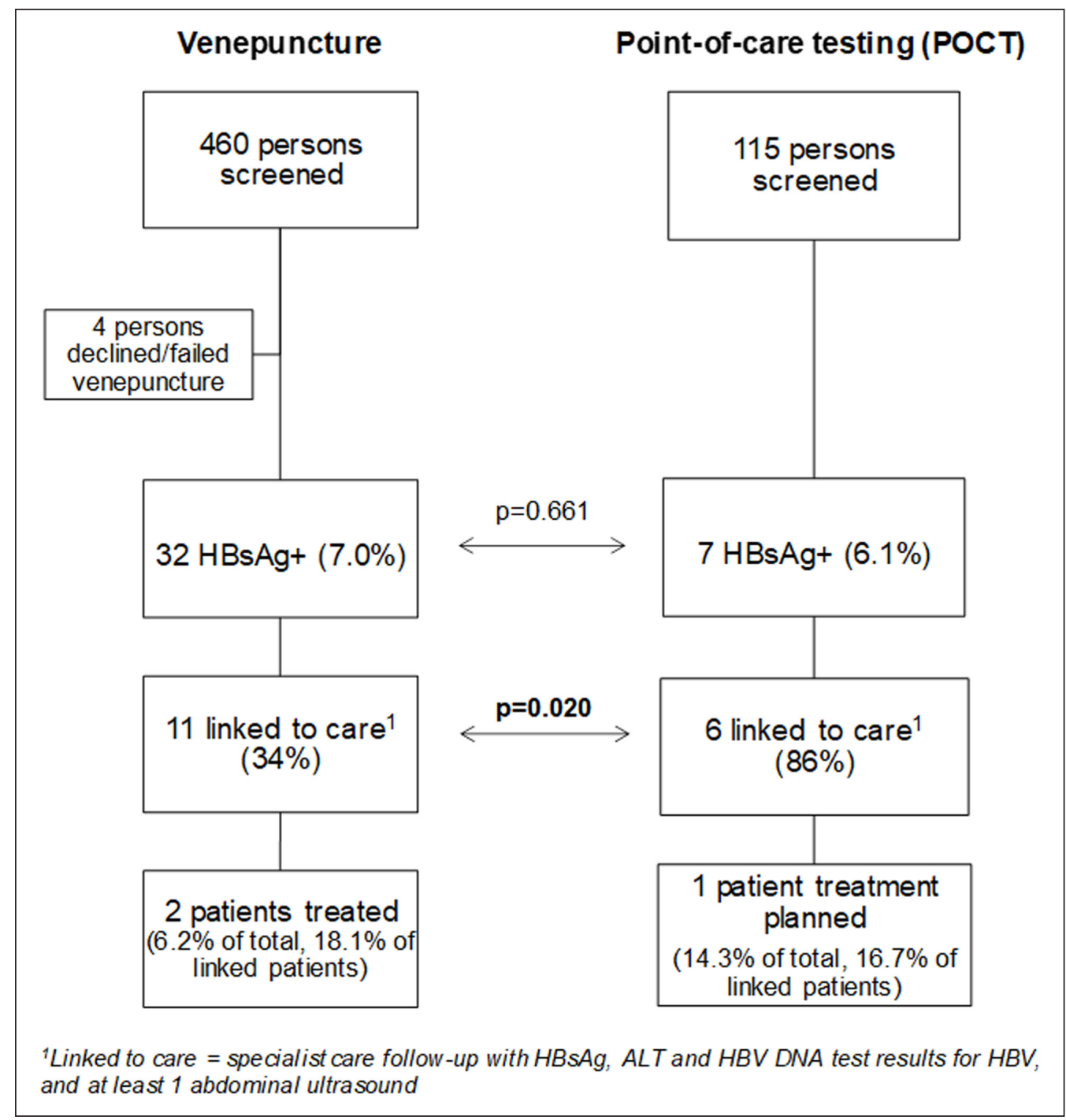

Figure 1: Overview of linkage to care of community screening using venepuncture and point-of-care testing (POCT).

\section{Epidemiology, Demographics}

In total, 575 persons participated, and 571 individuals were serologically screened. Using venepuncture, 456 persons had valid serologic results, and 115 persons were screened using POC testing. Overall, seroprevalence for HBsAg was $6.8 \%(39 / 571)$, with 32 and 7 individuals testing positive by venepuncture and POCT respectively. There was no statistically significant difference in HBsAg seroprevalence in both screening methods $(7.0 \%$ vs $6.1 \%$, $\mathrm{p}=0.661)$. An overview is shown in Figure 1. One person who tested positive for HBsAg was a second-generation migrant. This person was born before 1999, before universal vaccination in Belgium and also before generalised HBsAg pregnancy testing in 1982.

\section{Impact of point-of-care testing: comparison of} linkage to care, cost

The average turnaround time was 32 days (20-45 days, Figure 2) for venepuncture samples. Results were immediately available with POC testing. Eleven of the $32 \mathrm{HBsAg}$ positives diagnosed using venepuncture (34\%) were linked to specialist care, compared to six of the seven posi- 


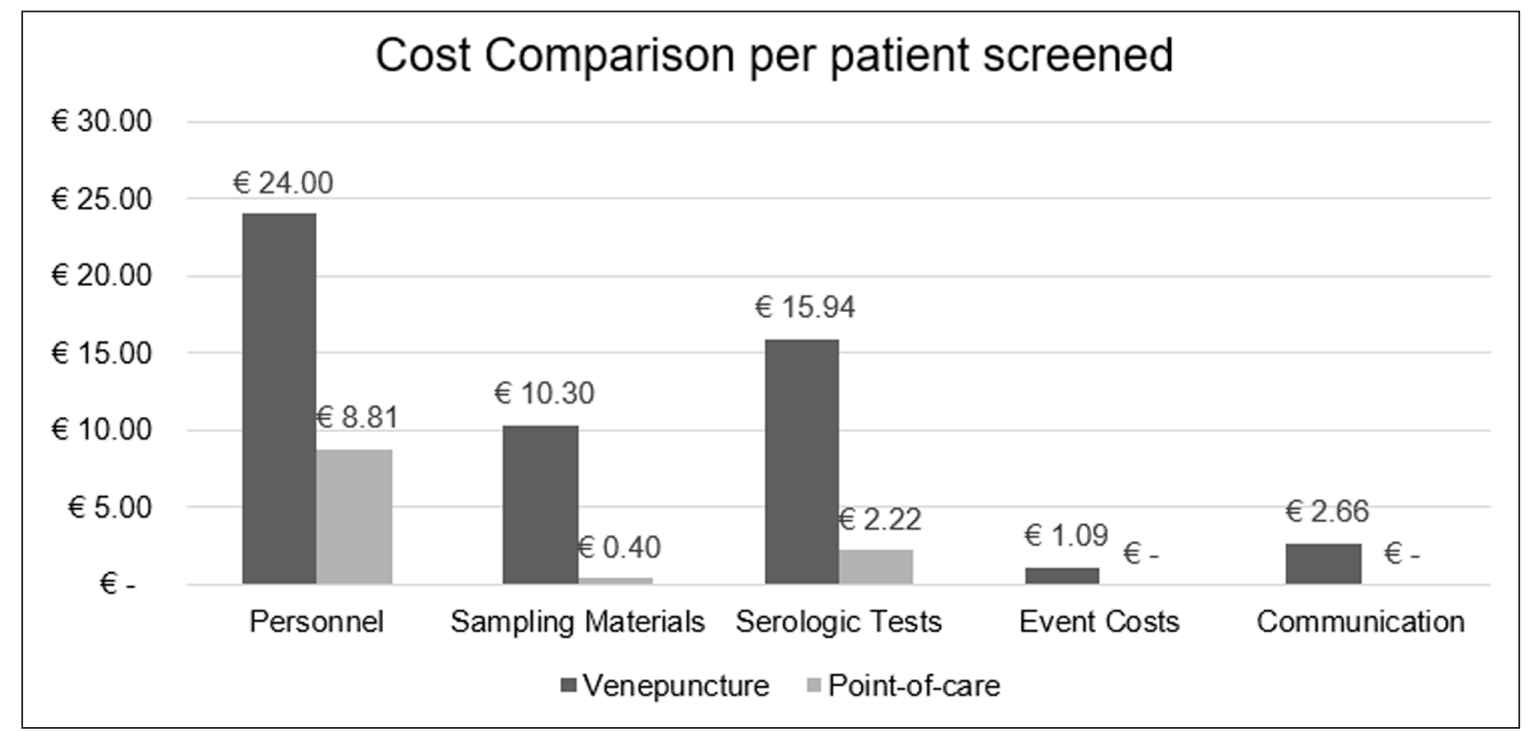

Figure 2: Turn-around time (time-to-result in days) for results of venepuncture screening.

Table 2: Characteristics of screened persons not linked to care/linked to care.

All $(n=39) \quad$ Not linked to care $^{1}(n=22) \quad$ Linked to care $(n=17) \quad$ p Value

\section{Demographics}

Age (mean, years) ${ }^{2}$

Gender (female, \%)

Country of origin (China, \%)

Residence (Antwerp, \%)

Type of screening (POCT, 6 \%)

Liver disease

HCC surveillance indication (yes, \%) ${ }^{2}$

Significant liver fibrosis 4

HBV clinical phase ${ }^{3}$

$$
\text { ALT > ULN }(\%)^{5}
$$

HBV DNA $>2,000 \mathrm{lU} / \mathrm{mL}(\%)$

HBV DNA > 20,000 IU/mL (\%)

HBeAg $(\%)$
$18(50.0)$

$6(16.7)$

3 (13.6)

46.5

22 (56.4)

38 (97.4)

19 (48.7)

7 (17.9)

$8(22.2)$

13 (36.1)

7 (19.4)

2 (5.1)
46.8

12 (54.5)

22 (100)

10 (45.5)

$1(4.5)$

10 (47.6)

3 (13.6)

$-$

$4(19.0)$

7 (33.3)

3 (14.3)
45.1

$10(58.8)$

16 (94.1)

9 (52.9)

$6(35.3)$

8 (44.4)

3 (16.7)

3 (16.7)

$4(26.7)$

0.59

$6(42.9)$

4 (28.6)

2 (11.8)

${ }^{1}$ Linked to care $=$ specialist care follow-up with HBsAg, ALT and HBV DNA test results and at least 1 abdominal ultrasound.

${ }^{2,3}$ Data available for $90 \%$ and $92 \%$ of patierns, respectively.

${ }^{4}$ detected using shear wave elastography, $6 \mathrm{kPa}$ or above [25].

${ }^{5}$ Upper Limit of Normal ALT: $41 \mathrm{U} / \mathrm{L}$ for males, $31 \mathrm{U} / \mathrm{L}$ for females.

${ }^{6}$ POCT: Point-of-care testing.

tive patients diagnosed with POC testing (86\%) (Figure 1; $\mathrm{p}=0.020$ ). As we collected demographic data in the total venepuncture cohort after informed consent and in those linked to care for POCT, we were able to analyse differences in demographics between patients linked to care in both programs, as well as those not-linked to care in the venepuncture program: Age, gender, country of origin and place of residence were not associated with linkage to care, or the absence thereof in the venepuncture program
(Table 2). There was no difference in the proportion of patients who met HCC surveillance criteria or treatment indications. Nor was there a difference in ALT and HBV DNA elevation between linked and non-linked patients in the venepuncture program. Ten $(25.6 \% ; 10 / 39)$ and three $(7.7 \% ; 3 / 39)$ of identified HBV patients met criteria for HCC surveillance or treatment initiation respectively [23] but were lost to follow-up (Table 2). Two patients screened with venepuncture are currently being treated 
Asian Migrant Population

with antiviral therapy according to their natural history and international treatment recommendations. For one patient screened with POC testing, antiviral treatment is being planned after a liver biopsy [21, 22]. None of the patients who were linked to care tested positive for antiHepatitis $C$ antibodies or anti-hepatitis Delta antibodies.

The costs associated with screening are shown in detail in Table 3.

Overall, the costs for screening were $€ 24,819$ and $€ 2,750$ for venepuncture and POC testing respectively. Taking into account the number of persons screened, the screening cost per person is $€ 54.0$ and $€ 11.4$ for venepuncture and POC testing respectively (Figure $\mathbf{3}$ ). Screening uptake differed between the two screening methods. While screening uptake was $100 \%$ when screening with venepuncture, uptake was $88.8 \%$ when using POC testing. Taking this into account, per person screening cost of the POC testing is $€$ 12.7. Total adjusted cost saving is $€ 41.3$ or $76.5 \%$.

\section{Discussion}

This study provides empirical proof that POC screening tests for viral hepatitis $B$ are associated with a higher linkage to care in an Asian migrant population, despite a lower overall cost and lower cost per person screened.

The WHO recently launched its ambition to eliminate viral hepatitis $B$ and $C$ infections as a public health problem by 2030 [3]. Although diagnostic tests with highperformance characteristics $[14,15]$ and effective antiviral treatments are available, attaining the ambitious WHO goal will depend on identifying currently unknown carriers and linking them to specialist care. Linkage to care remains suboptimal, certainly in populations where viral hepatitis is highly endemic. In our data, a pressing sign of the importance of linkage to care is that $7.7 \%(n=3 / 39)$ and $25.6 \%(n=10 / 39)$ of the identified HBV patients who had treatment and HCC surveillance indications respectively in the venepuncture program [22], were lost to follow-up (Table 2).

Interestingly, a recent study by Sehr et al [19]. used Markov modelling to predict that POC testing may decrease long-term complications due to chronic HBV infection and improve linkage to care. Our data empirically support the wider implementation of POC testing due to its higher linkage to care of $86 \%$ of identified patients.

Only one-third of HBsAg positive individuals were linked to care using venepuncture-based testing, a result corroborated by numerous other studies $[9,11,17,18]$. Multiple barriers towards screening and linkage to care in regards to viral hepatitis exist in the Chinese community. A recent qualitative study by Lee et al [12]. highlights determinants in knowledge, cultural beliefs, social stigma, awareness, and views towards Western health systems as major contributors as to why viral hepatitis is an "invisible disease in an invisible population". Barriers to care have also been investigated in other populations and HCV, HIV, and testing of sexually transmissible diseases $[8,13]$. Linkage to care was enhanced by facilitating referral for $\mathrm{HCV}$ assessment and scheduling of specialist appointments for screened persons. Barriers described in HIV testing included transport costs and distance, stigma, and fear of disclosure-factors similar to those described in screening for viral hepatitis in Asian populations.

Finally, we observed an overall HBsAg seroprevalence of $6.8 \%$ in the Asian, predominantly Chinese migrant community in three major cities in Belgium, which is in line with similar studies from the UK and the Netherlands [5-7].

Many potential issues and limitations must be addressed in our study.

Table 3: Overall costs of venepuncture and point-of-care testing (POCT) screening (in euros).

\begin{tabular}{llrr} 
& & Venepuncture & \multicolumn{1}{c}{ POC } \\
\hline Personnel & Nursing staff & 3634.0 & 0.0 \\
& Administrative assistant & 1157.7 & 0.0 \\
& Language services & 160.0 & 0.0 \\
& Study coordinator & 1719.8 & 1013.5 \\
& Physicians & 4366.8 & 0.0 \\
& TOTAL & 11038.3 & 1013.5 \\
Logistics & Blood tubes, venepuncture materials & 4737.2 & 46.2 \\
& Serological tests: HBsAg & 7332.5 & 254.9 \\
& Event logistics (location rent, catering, etc) & 500.0 & 0.0 \\
& Communication costs & 1211.0 & 0.0 \\
& TOTAL & 13780.7 & 301.1 \\
OVERALL COST & & 24819.0 & 1314.6 \\
Screening uptake & & $100 \%$ & $88.8 \%$ \\
ADJUSTED COST & & $\mathbf{2 4 8 1 9 . 0}$ & $\mathbf{1 4 6 1 . 8}$
\end{tabular}




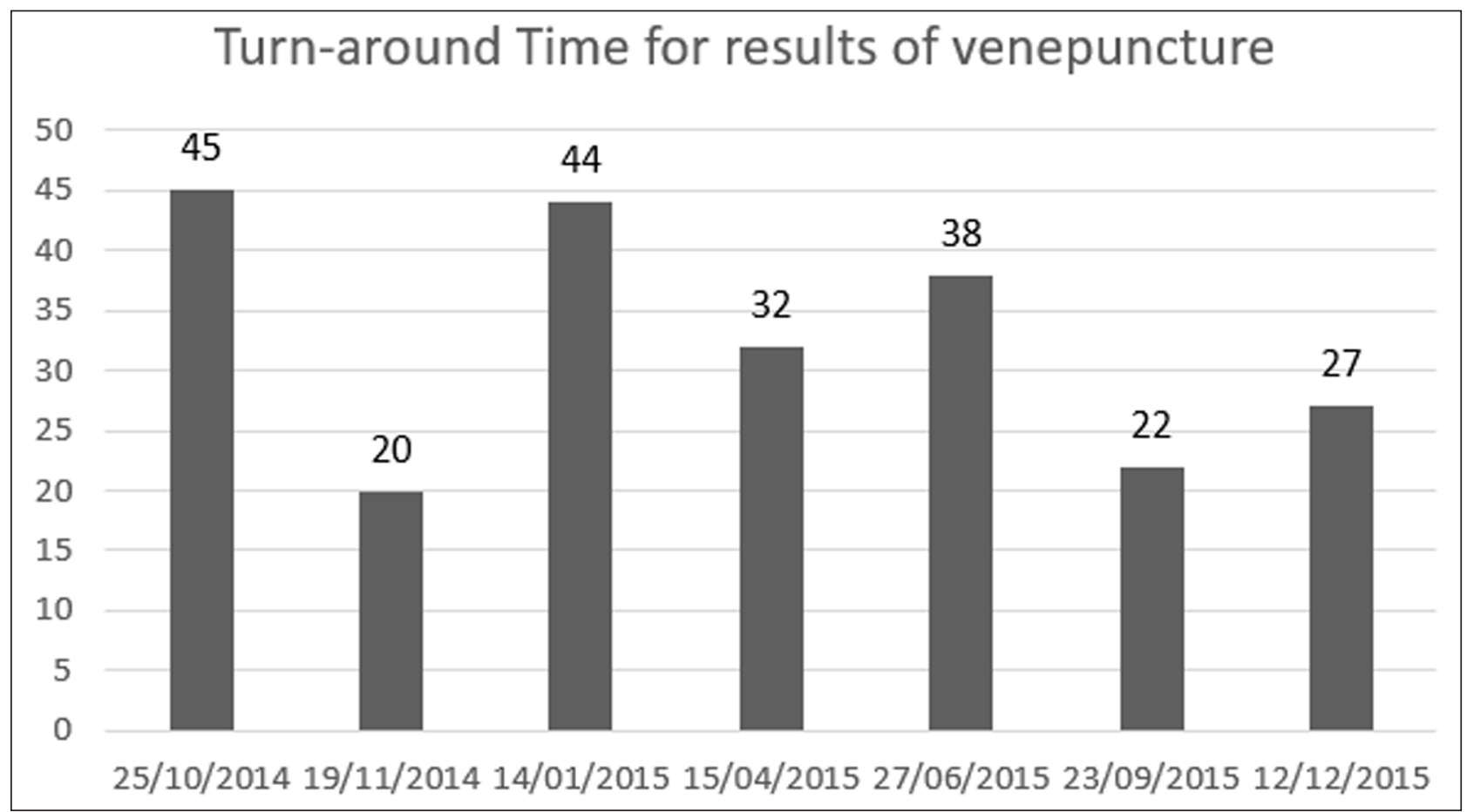

Figure 3: Cost comparison per patient screened (in euros).

First, it could be argued that the samples of both screening methods are not the same: venepuncture and POC tests were not randomised side by side at each screening site. However, the lack of statistical difference in seroprevalence of HBsAg between both protocols provides indirect evidence on the contrary. Also, when observing screened persons' characteristics as a whole and comparing positively screened individuals based on their linkage to care (Table 2), all described characteristics showed no statistically significant difference across both groups except the screening method used $(\mathrm{p}=0.020)$.

Second, while this study focused on the effect of POC testing on linkage to care, other factors besides the test modality differed between screening protocols. During venepuncture screening, persons were specifically motivated by community leaders and through peers to get tested. This may explain the observed higher screening uptake. POC testing was mostly an opportunistic screening during classes/professional activities. As opportunistic screening generally has a lower impact [23], the higher linkage to care during POC testing further underlines the importance of providing on-site results to motivate patients for clinical follow-up.

Finally, an a priori power analysis for linkage to care was not possible. Using observed data, however, the estimated power surpasses $80 \%$. The high prevalence of HBV in this population and their well-known low rate of linkage to care allowed investigating the impact of POCT on linkage to care. Such an impact would be harder to assess in Caucasians living in a low seroprevalence area and with generally higher linkage to care [20].

\section{Conclusion}

In an Asian population with a high HBV prevalence, we found that screening based on POC tests results in lower costs per person screened (76.5\%), and a 2.5 times higher linkage to care.

\section{Data Accessibility Statement}

The (anonymized) datasets used and/or analysed during the current study are available from the corresponding author on reasonable request.

\section{Ethics and Consent}

The screening programs have been approved by the Ethical Committee of the Antwerp University Hospital/Antwerp University (B300201421693, B300201629236).

\section{Acknowledgements}

All Chinese community leaders: Pastor Andrew Chan, Ms. Elaine Yan, Ms. Vivianne Lo, Pastor Timothy Tang, Mrs. Judy Cui, Mrs. Anna Tang, Ms. Liu Yee Wan, all Chinese community translators and volunteers, City Council Antwerp, (study) nurses, and all Antwerp University Hospital and Antwerp University personnel.

\section{Funding Information}

This study was performed with grants from the following institutions and companies:

\section{Roche Diagnostics}

Gilead Life Sciences Inc.

Bristol-Myers Squibb

Sandoz

Centre for Medical Innovation - Authorities of the Region of Flanders

Thomas Vanwolleghem is supported by a Research Mandate of the Foundation Against Cancer Belgium, No. 2014-087. 


\section{Competing Interests}

The authors have no competing interests to declare.

\section{Author Contributions}

- EH compiled and analysed the data, drafted the manuscript, and is the corresponding author.

- PM contributed to the coordination and execution of screening events, provided data, and co-drafted the manuscript.

- PVD co-drafted the manuscript and guided the overall coordination of the project

- MI provided oversight and advice in laboratory/invitro testing.

- IV contributed to the statistical analysis of the data, the overall direction of the study in terms of study design and execution, and co-drafted the manuscript.

- TV is the senior author and contributed to the screening events, data collection, the overall direction of the study, and co-drafted the manuscript.

- All authors read and approved the final manuscript.

\section{References}

1. World Health Organization. WHO Global Hepatitis Report. World Health Organization; 2017.

2. Moraga, P. GBD 2016 Causes of Death Collaborators Global, regional, and national age-sex specific mortality for 264 causes of death, 1980-2016: A systematic analysis for the Global Burden of Disease Study 2016. Lancet. 2017; 390(10100): 1151-1210.

3. World Health Organization. Advocacy brief. Combating Hepatitis $B$ and $C$ to reach elimination by 2030. World Health Organization; 2016.

4. World Health Organization. Guidelines on hepatitis B and C testing. New evidence-based global guidelines on who to test and how to test for chronic hepatitis B and C, and strategies for implementation. World Health Organization; 2017.

5. Vedio AB, Ellam H, Rayner F. Hepatitis B: Report of prevalence and access to healthcare among Chinese residents in Sheffield UK. J Infect Public Health. 2013; 6(6): 448-55. DOI: https://doi. org/10.1016/j.jiph.2013.05.004

6. Veldhuijzen IK, Wolter R, Rijckborst V. Identification and treatment of chronic hepatitis B in Chinese migrants: Results of a project offering on-site testing in Rotterdam, The Netherlands. J Hepatol. 2012; 57(6): 1171-6. DOI: https://doi.org/10.1016/j. jhep.2012.07.036

7. Cochrane A, Evlampidou I, Irish C. Hepatitis B infection prevalence by country of birth in migrant populations in a large UK city. J Clin Virol. 2015; 68: 79-82. DOI: https://doi.org/10.1016/j.jcv.2015.05.009

8. Perumalswami, Ponni V, Stephanie H. Hepatitis Outreach Network: A practical strategy for hepatitis screening with linkage to care in foreign-born communities. Journal of hepatology. 2013; 58(5):890-897. DOI: https://doi.org/10.1016/j.jhep.2013.01.004

9. Perumalswami, Ponni V, Stephanie H. Hepatitis Outreach Network: A practical strategy for hepatitis screening with linkage to care in foreign-born communities. Journal of Hepatology. 2013; 58(5): 890-897. DOI: https://doi.org/10.1016/j. jhep.2013.01.004

10. Hahne SJ, Veldhuijzen IK. Infection with hepatitis $B$ and $C$ virus in Europe: A systematic review of prevalence and cost-effectiveness of screening. BMC Infect Dis. 2013; 13: 181. DOI: https://doi. org/10.1186/1471-2334-13-181

11. Harris AM, Schoenbachler BT. Testing and linking foreign-born people with chronic hepatitis B virus infection to care at nine U.S. programs, 2012-2014. Public Health Rep. 2016; 131(2): 20-8. DOI: https:// doi.org/10.1177/00333549161310S204

12. Lee ACK, Vedio A. Determinants of uptake of hepatitis $B$ testing and healthcare access by migrant Chinese in the England: A qualitative study. $B M C$ Public Health. 2017; 17(1): 747. DOI: https://doi. org/10.1186/s12889-017-4796-4

13. Govindasamy D1, Ford N. Risk factors, barriers and facilitators for linkage to antiretroviral therapy care: A systematic review. AIDS. 2012; 26(16): 2059-67. DOI: https://doi.org/10.1097/ QAD.0b013e3283578b9b

14. Scalioni Lde P, Cruz HM, de Paula VS. Performance of rapid hepatitis $C$ virus antibody assays among high- and low-risk populations. J Clin Virol. 2014; 60(3): 200-5. DOI: https://doi.org/10.1016/j. jcv.2014.04.001

15. Bottero J, Boyd A, Gozlan J. Performance of rapid tests for detection of HBsAg and anti-HBsAb in a large cohort. France. J Hepatol. 2013; 58(3): 473-8. DOI: https://doi.org/10.1016/j.jhep.2012.11.016

16. Chinese Migration to Belgium - Trends and Perspectives. Myria, Belgian Federal Migration Centre. 2005.

17. Stanford J, Biba A. Community-engaged strategies to promote hepatitis B testing and linkage to care in immigrants of Florida. J Epidemiol Glob Health. 2016; 6(4): 277-284. DOI: https://doi. org/10.1016/j.jegh.2016.06.003

18. Hyun CS, Kim S. Chronic hepatitis B in Korean Americans: Decreased prevalence and poor linkage to care. BMC Infect Dis. 2016; 16(1): 415. DOI: https://doi.org/10.1186/s12879-016-1732-7

19. Sehr MA, Joshi KD. Markov modeling in hepatitis B screening and linkage to care. Theor Biol Med Model. 2017; 14(1): 11. DOI: https://doi.org/10.1186/ s12976-017-0057-6

20. Janjua NZ, Kuo M, Chong M. Assessing hepatitis $\mathrm{C}$ burden and treatment effectiveness through the British Columbia Hepatitis Testers Cohort (BC-HTC): Design and characteristics of linked and unlinked participants. PLoS One. 2016; 11(3): e0150176. DOI https://doi.org/10.1371/journal.pone.0150176

21. Vanwolleghem T, Hou J, van Oord G. Re-evaluation of hepatitis B virus clinical phases by systems biology identifies unappreciated roles for the innate immune response and B cells. Hepatology. 2015; 62(1): 87-100. DOI: https://doi.org/10.1016/ S0168-8278(15)30664-4 Epub 2015 Apr 27. 
22. European Association for the Study of the Liver. EASL 2017 Clinical Practice Guidelines on the management of hepatitis B virus infection. $J$ Hepatol. 2017; 67(2): 370-398. DOI: https://doi. org/10.1016/j.jhep.2017.03.021

23. Robotin MC, George J. Community-based hepatitis B screening: What works? Hepatol Int. 2014; 8(4): 478-92. DOI: https://doi.org/10.1007/ s12072-014-9562-4

24. European Association for the Study of the Liver, European Organisation for Research
And Treatment of Cancer. EASL-EORTC clinical practice guidelines: Management of hepatocellular carcinoma. J Hepatol. 2012; 56(4): 908-43. DOI: https://doi.org/10.1016/j.jhep.2011.1 2.001

25. The French METAVIR Cooperative Study Group. Intraobserver and interobserver variations in liver biopsy interpretation in patients with chronic hepatitis C. Hepatology. 1994; 20(1 Pt 1): 15-20. DOI: https://doi.org/10.1002/hep.1840200 104

How to cite this article: Ho E, Michielsen P, Van Damme P, leven M, Veldhuijzen I, Vanwolleghem T. Point-of-Care Tests for Hepatitis B Are Associated with A Higher Linkage to Care and Lower Cost Compared to Venepuncture Sampling During Outreach Screenings in an Asian Migrant Population. Annals of Global Health. 2020; 86(1): 81, 1-8. DOl: https://doi.org/10.5334/ aogh.2848

Published: 16 July 2020

Copyright: (c) 2020 The Author(s). This is an open-access article distributed under the terms of the Creative Commons Attribution 4.0 International License (CC-BY 4.0), which permits unrestricted use, distribution, and reproduction in any medium, provided the original author and source are credited. See http://creativecommons.org/licenses/by/4.0/. 